\title{
Efficient Fast Multiplication Free Integer Transformation for the 1-D DCT of the H.265 Standard
}

\author{
Mohamed Nasr Haggag ${ }^{1}$, Mohamed El-Sharkawy ${ }^{2}$, Gamal Fahmy $^{3}$, Maher Rizkalla ${ }^{1}$ \\ ${ }^{1}$ German University at Cairo New Cairo City, Cairo, Egypt; ${ }^{2}$ Egypt Japan University of Science and Technology Borg Al Arab, \\ Alexandria, Egypt; ${ }^{3}$ Electrical Engineering Department, Assiut University, Assiut, Egypt. \\ Email: mrizkall@iupui.edu
}

Received January $21^{\text {st }} 2010$; revised June $30^{\text {th }} 2010$; accepted July $5^{\text {th }} 2010$.

\begin{abstract}
In this paper, efficient one-dimensional (1-D) fast integer transform algorithms of the DCT matrix for the H.265 standard is proposed. Based on the symmetric property of the integer transform matrix and the matrix operations, which denote the row/column permutations and the matrix decompositions, along with using the dyadic symmetry modification on the standard matrix, the efficient fast 1-D integer transform algorithms are developed. Therefore, the computational complexities of the proposed fast integer transform are smaller than those of the direct method. In addition to computational complexity reduction one of the proposed algorithms provides transformation quality improvement, while the other provides more computational complexity reduction while maintaining almost the same transformation quality. With lower complexity and better transformation quality, the first proposed fast algorithm is suitable to accelerate the quality-demanding video coding computations. On the other hand, with the significant lower complexity, the second proposed fast algorithm is suitable to accelerate the video coding computations.
\end{abstract}

Keywords: Fast Algorithm, HDTV, H.265, ICT, Order-16 Transform, Video Coding

\section{Introduction}

NOWDAYS the demand for higher quality digital video products and faster digital video applications in our daily life activities is increasing. These demands start from our daily necessary needs, like video conferencing, television and surveillance, up to our entertainment, iPods, internet video streaming, digital cameras, and all high definition (HD) products $[1,2]$. There have been two primary standards organizations driving the definition of video coding. The International Telecommunications Union (ITU), which is an organization focused on telecommunication applications and has created the series of H.26x standards for low bit rate video telephony. These include H.261, H.262, H.263 and H.264. The other organization is the International Standards Organization (ISO), which is more focused on consumer applications and has defined the MPEG series standards for compressing moving pictures. The MPEG standard series include MPEG-1, MPEG-2 and MPEG-4 [1,2].

In [3], the $16 \times 16$ 2-D matrix for the H.265 standard DCT is revealed, using the decomposition and the mod- ification techniques used in [4-7], this paper will introduce two proposed algorithm that will aim to reduce the complexity of the algorithm implementation in addition to making it multiplication-free.

The rest of this paper is organized as follows. In Section 2, review of the integer transformation for the H.265 standard is described. In Section 3, the two proposed efficient fast integer transform algorithms for the 2-D H.265 standard are introduced with the proposed matrix factorizations. Then the computational complexities of these proposed algorithms are discussed. In Section 4, analysis and comparison of transformation quality between the proposed fast algorithms and the original method is shown. In Section 5, comparison of computational complexity done in Section 3 and quality evaluation done in Section 4 is discussed. Finally, we give a conclusion.

\section{Review of the Integer Transformation for the H.265 Standard}

The H.265 is the newest yet to be released standard for 
high definition video processing. From [3], the matrix of the 2-D $16 \times 16$ integer cosine transformation for the H.265 standard is shown in Equation (1).

The matrix elements in Equation (1) shows that there is symmetric properties between the left side and right side of the matrix, this property will be exploited using matrix decomposition in order to this matrix into the product of sparse matrices in the next section.

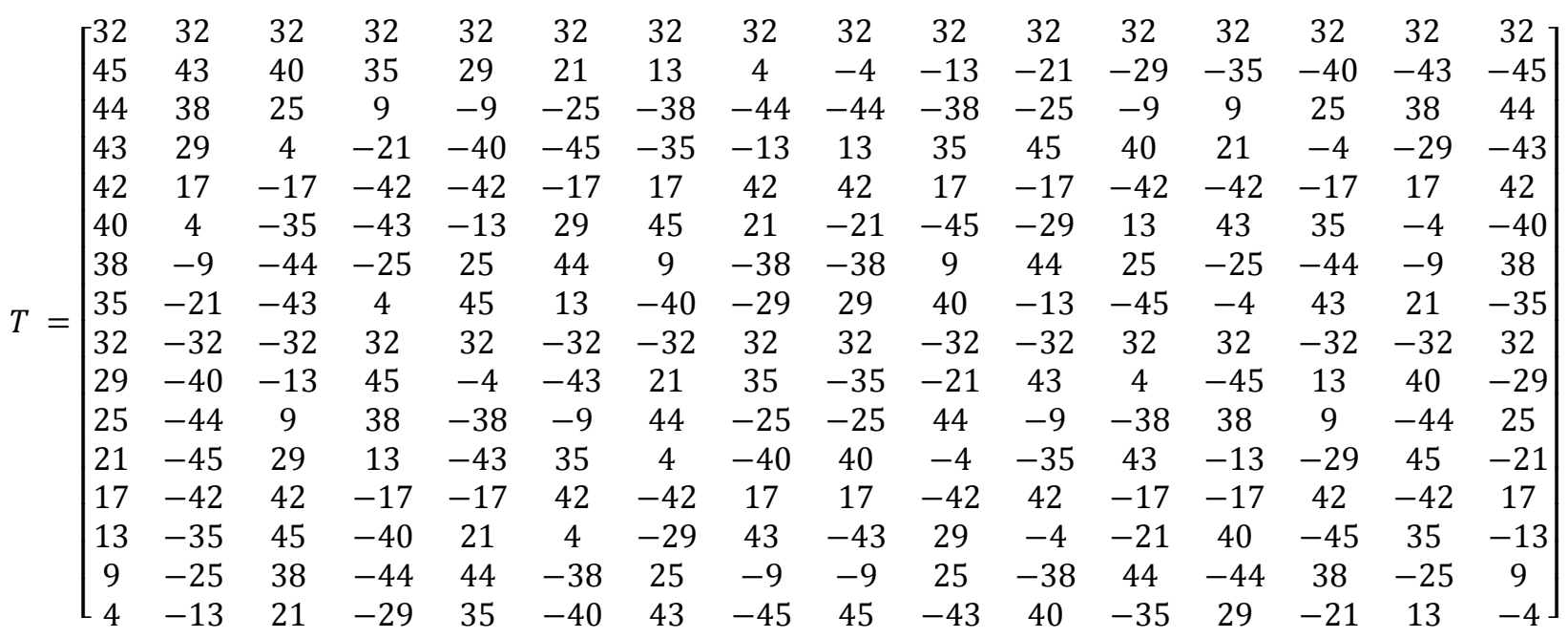

\section{Proposed Algorithms}

In this section, two proposed algorithm for efficient fast multiplication-free for the H.265 standard are presented. The proposed algorithms are done using a combination of Modified Integer Cosine Transformation, matrix decomposition and dyadic symmetry. The common part in the complexity reduction is discussed first, then each algorithm is presented individually and its complexity is calculated with it. The aim of the proposed algorithms is to reduce the computational complexities, which are refe- rred to as the numbers of additions and shift operations as much as possiblewhile maintaining reasonable error margin. The DCT matrix for the H.265 is given as $\boldsymbol{T}$ in Equation (1).

The symmetric property of the transformation matrix is exploited to decompose it into the product of two sparse matrices. so the transformation matrix in Equation 1 can be rewritten in Equation (2) [4,7].

$$
T=T_{T} \cdot P_{1}
$$

$$
T_{T}=\left[\begin{array}{cccccccccccccccc}
32 & 32 & 32 & 32 & 32 & 32 & 32 & 32 & 0 & 0 & 0 & 0 & 0 & 0 & 0 & 0 \\
0 & 0 & 0 & 0 & 0 & 0 & 0 & 0 & -4 & -13 & -21 & -29 & -35 & -40 & -43 & -45 \\
44 & 38 & 25 & 9 & -9 & -25 & -38 & -44 & 0 & 0 & 0 & 0 & 0 & 0 & 0 & 0 \\
0 & 0 & 0 & 0 & 0 & 0 & 0 & 0 & 13 & 35 & 45 & 40 & 21 & -4 & -29 & -43 \\
42 & 17 & -17 & -42 & -42 & -17 & 17 & 42 & 0 & 0 & 0 & 0 & 0 & 0 & 0 & 0 \\
0 & 0 & 0 & 0 & 0 & 0 & 0 & 0 & -21 & -45 & -29 & 13 & 43 & 35 & -4 & -40 \\
38 & -9 & -44 & -25 & 25 & 44 & 9 & -38 & 0 & 0 & 0 & 0 & 0 & 0 & 0 & 0 \\
0 & 0 & 0 & 0 & 0 & 0 & 0 & 0 & 29 & 40 & -13 & -45 & -4 & 43 & 21 & -35 \\
32 & -32 & -32 & 32 & 32 & -32 & -32 & 32 & 0 & 0 & 0 & 0 & 0 & 0 & 0 & 0 \\
0 & 0 & 0 & 0 & 0 & 0 & 0 & 0 & -35 & -21 & 43 & 4 & -45 & 13 & 40 & -29 \\
25 & -44 & 9 & 38 & -38 & -9 & 44 & -25 & 0 & 0 & 0 & 0 & 0 & 0 & 0 & 0 \\
0 & 0 & 0 & 0 & 0 & 0 & 0 & 0 & 40 & -4 & -35 & 43 & -13 & -29 & 45 & -21 \\
17 & -42 & 42 & -17 & -17 & 42 & -42 & 17 & 0 & 0 & 0 & 0 & 0 & 0 & 0 & 0 \\
0 & 0 & 0 & 0 & 0 & 0 & 0 & 0 & -43 & 29 & -4 & -21 & 40 & -45 & 35 & -13 \\
9 & -25 & 38 & -44 & 44 & -38 & 25 & -9 & 0 & 0 & 0 & 0 & 0 & 0 & 0 & 0 \\
0 & 0 & 0 & 0 & 0 & 0 & 0 & 0 & 45 & -43 & 40 & -35 & 29 & -21 & 13 & -4
\end{array}\right]
$$




$$
P_{1}=\left[\begin{array}{cccccccccccccccc}
1 & 0 & 0 & 0 & 0 & 0 & 0 & 0 & 0 & 0 & 0 & 0 & 0 & 0 & 0 & 1 \\
0 & 1 & 0 & 0 & 0 & 0 & 0 & 0 & 0 & 0 & 0 & 0 & 0 & 0 & 1 & 0 \\
0 & 0 & 1 & 0 & 0 & 0 & 0 & 0 & 0 & 0 & 0 & 0 & 0 & 1 & 0 & 0 \\
0 & 0 & 0 & 1 & 0 & 0 & 0 & 0 & 0 & 0 & 0 & 0 & 1 & 0 & 0 & 0 \\
0 & 0 & 0 & 0 & 1 & 0 & 0 & 0 & 0 & 0 & 0 & 1 & 0 & 0 & 0 & 0 \\
0 & 0 & 0 & 0 & 0 & 1 & 0 & 0 & 0 & 0 & 1 & 0 & 0 & 0 & 0 & 0 \\
0 & 0 & 0 & 0 & 0 & 0 & 1 & 0 & 0 & 1 & 0 & 0 & 0 & 0 & 0 & 0 \\
0 & 0 & 0 & 0 & 0 & 0 & 0 & 1 & 1 & 0 & 0 & 0 & 0 & 0 & 0 & 0 \\
0 & 0 & 0 & 0 & 0 & 0 & 0 & -1 & 1 & 0 & 0 & 0 & 0 & 0 & 0 & 0 \\
0 & 0 & 0 & 0 & 0 & 0 & -1 & 0 & 0 & 1 & 0 & 0 & 0 & 0 & 0 & 0 \\
0 & 0 & 0 & 0 & 0 & -1 & 0 & 0 & 0 & 0 & 1 & 0 & 0 & 0 & 0 & 0 \\
0 & 0 & 0 & 0 & -1 & 0 & 0 & 0 & 0 & 0 & 0 & 1 & 0 & 0 & 0 & 0 \\
0 & 0 & 0 & -1 & 0 & 0 & 0 & 0 & 0 & 0 & 0 & 0 & 1 & 0 & 0 & 0 \\
0 & 0 & -1 & 0 & 0 & 0 & 0 & 0 & 0 & 0 & 0 & 0 & 0 & 1 & 0 & 0 \\
0 & -1 & 0 & 0 & 0 & 0 & 0 & 0 & 0 & 0 & 0 & 0 & 0 & 0 & 1 & 0 \\
-1 & 0 & 0 & 0 & 0 & 0 & 0 & 0 & 0 & 0 & 0 & 0 & 0 & 0 & 0 & 1
\end{array}\right]
$$

Where

$$
T_{T}=P_{r} \cdot T_{r}
$$

$$
\operatorname{Pr}=\left[\begin{array}{llllllllllllllll}
1 & 0 & 0 & 0 & 0 & 0 & 0 & 0 & 0 & 0 & 0 & 0 & 0 & 0 & 0 & 0 \\
0 & 0 & 0 & 0 & 0 & 0 & 0 & 0 & 1 & 0 & 0 & 0 & 0 & 0 & 0 & 0 \\
0 & 1 & 0 & 0 & 0 & 0 & 0 & 0 & 0 & 0 & 0 & 0 & 0 & 0 & 0 & 0 \\
0 & 0 & 0 & 0 & 0 & 0 & 0 & 0 & 0 & 1 & 0 & 0 & 0 & 0 & 0 & 0 \\
0 & 0 & 1 & 0 & 0 & 0 & 0 & 0 & 0 & 0 & 0 & 0 & 0 & 0 & 0 & 0 \\
0 & 0 & 0 & 0 & 0 & 0 & 0 & 0 & 0 & 0 & 1 & 0 & 0 & 0 & 0 & 0 \\
0 & 0 & 0 & 1 & 0 & 0 & 0 & 0 & 0 & 0 & 0 & 0 & 0 & 0 & 0 & 0 \\
0 & 0 & 0 & 0 & 0 & 0 & 0 & 0 & 0 & 0 & 0 & 1 & 0 & 0 & 0 & 0 \\
0 & 0 & 0 & 0 & 1 & 0 & 0 & 0 & 0 & 0 & 0 & 0 & 0 & 0 & 0 & 0 \\
0 & 0 & 0 & 0 & 0 & 0 & 0 & 0 & 0 & 0 & 0 & 0 & 1 & 0 & 0 & 0 \\
0 & 0 & 0 & 0 & 0 & 1 & 0 & 0 & 0 & 0 & 0 & 0 & 0 & 0 & 0 & 0 \\
0 & 0 & 0 & 0 & 0 & 0 & 0 & 0 & 0 & 0 & 0 & 0 & 0 & 1 & 0 & 0 \\
0 & 0 & 0 & 0 & 0 & 0 & 1 & 0 & 0 & 0 & 0 & 0 & 0 & 0 & 0 & 0 \\
0 & 0 & 0 & 0 & 0 & 0 & 0 & 0 & 0 & 0 & 0 & 0 & 0 & 0 & 1 & 0 \\
0 & 0 & 0 & 0 & 0 & 0 & 0 & 1 & 0 & 0 & 0 & 0 & 0 & 0 & 0 & 0 \\
0 & 0 & 0 & 0 & 0 & 0 & 0 & 0 & 0 & 0 & 0 & 0 & 0 & 0 & 0 & 1
\end{array}\right]
$$

and

$$
T_{r}=\left[\begin{array}{cccccccccccccccc}
32 & 32 & 32 & 32 & 32 & 32 & 32 & 32 & 0 & 0 & 0 & 0 & 0 & 0 & 0 & 0 \\
44 & 38 & 25 & 9 & -9 & -25 & -38 & -44 & 0 & 0 & 0 & 0 & 0 & 0 & 0 & 0 \\
42 & 17 & -17 & -42 & -42 & -17 & 17 & 42 & 0 & 0 & 0 & 0 & 0 & 0 & 0 & 0 \\
38 & -9 & -44 & -25 & 25 & 44 & 9 & -38 & 0 & 0 & 0 & 0 & 0 & 0 & 0 & 0 \\
32 & -32 & -32 & 32 & 32 & -32 & -32 & 32 & 0 & 0 & 0 & 0 & 0 & 0 & 0 & 0 \\
25 & -44 & 9 & 38 & -38 & -9 & 44 & -25 & 0 & 0 & 0 & 0 & 0 & 0 & 0 & 0 \\
17 & -42 & 42 & -17 & -17 & 42 & -42 & 17 & 0 & 0 & 0 & 0 & 0 & 0 & 0 & 0 \\
9 & -25 & 38 & -44 & 44 & -38 & 25 & -9 & 0 & 0 & 0 & 0 & 0 & 0 & 0 & 0 \\
0 & 0 & 0 & 0 & 0 & 0 & 0 & 0 & -4 & -13 & -21 & -29 & -35 & -40 & -43 & -45 \\
0 & 0 & 0 & 0 & 0 & 0 & 0 & 0 & 13 & 35 & 45 & 40 & 21 & -4 & -29 & -43 \\
0 & 0 & 0 & 0 & 0 & 0 & 0 & 0 & -21 & -45 & -29 & 13 & 43 & 35 & -4 & -40 \\
0 & 0 & 0 & 0 & 0 & 0 & 0 & 0 & 29 & 40 & -13 & -45 & -4 & 43 & 21 & -35 \\
0 & 0 & 0 & 0 & 0 & 0 & 0 & 0 & -35 & -21 & 43 & 4 & -45 & 13 & 40 & -29 \\
0 & 0 & 0 & 0 & 0 & 0 & 0 & 0 & 40 & -4 & -35 & 43 & -13 & -29 & 45 & -21 \\
0 & 0 & 0 & 0 & 0 & 0 & 0 & 0 & -43 & 29 & -4 & -21 & 40 & -45 & 35 & -13 \\
0 & 0 & 0 & 0 & 0 & 0 & 0 & 0 & 45 & -43 & 40 & -35 & 29 & -21 & 13 & -4
\end{array}\right]
$$


The function of $\boldsymbol{P}_{\mathbf{1}}$ is the post-process matrix for the input data to the matrix multiplication, and the postprocess only uses the additions and subtracts. The computational complexities of $\boldsymbol{P}_{\mathbf{1}}$ are 16 additions. In Equation (2), the elements of $\boldsymbol{T}_{\boldsymbol{T}}$ are scattered; the rearrangement of the elements in $\boldsymbol{T}_{\boldsymbol{T}}$ is required to group the elements of $\boldsymbol{T}_{\boldsymbol{T}}$ into two $8 \times 8$ independent matrices. $\boldsymbol{P}_{\boldsymbol{r}}$ is a pre-process matrix that permutes the rows of $\boldsymbol{T}$, so that it is rewritten as in Equation (3) [4,7].

As shown in Equation (3), the matrix $\boldsymbol{P}_{\boldsymbol{r}}$ can be used as a pre-process matrix. $\boldsymbol{P}_{\boldsymbol{r}}$ doesn't have any complexity at all while serving the purpose of rearranging $\boldsymbol{T}_{\boldsymbol{T}}$ into $\boldsymbol{T}_{\boldsymbol{r}}$ where the matrix can be easily represented as the direct sum of its two non-zero areas. The result of the direct sum is shown in Equation (4).

$$
T_{r}=T_{00} \oplus T_{11}
$$

Where

$$
T_{00}=\left[\begin{array}{cccccccc}
32 & 32 & 32 & 32 & 32 & 32 & 32 & 32 \\
44 & 38 & 25 & 9 & -9 & -25 & -38 & -44 \\
42 & 17 & -17 & -42 & -42 & -17 & 17 & 42 \\
38 & -9 & -44 & -25 & 25 & 44 & 9 & -38 \\
32 & -32 & -32 & 32 & 32 & -32 & -32 & 32 \\
25 & -44 & 9 & 38 & -38 & -9 & 44 & -25 \\
17 & -42 & 42 & -17 & -17 & 42 & -42 & 17 \\
9 & -25 & 38 & -44 & 44 & -38 & 25 & -9
\end{array}\right]
$$

And

$$
T_{11}=\left[\begin{array}{cccccccc}
-4 & -13 & -21 & -29 & -35 & -40 & -43 & -45 \\
13 & 35 & 45 & 40 & 21 & -4 & -29 & -43 \\
-21 & -45 & -29 & 13 & 43 & 35 & -4 & -40 \\
29 & 40 & -13 & 45 & -4 & 43 & 21 & -35 \\
-35 & -21 & 43 & 4 & -45 & 13 & 40 & -29 \\
40 & -4 & -35 & 43 & -13 & -29 & 45 & -21 \\
-43 & 29 & -4 & -21 & 40 & -45 & 35 & -13 \\
45 & -43 & 40 & -35 & 29 & -21 & 13 & -4
\end{array}\right]
$$

The computation of $\boldsymbol{T}_{\mathbf{0 0}}$ can be called the computation of the even frequency part in the transform matrix, and the computation of $\boldsymbol{T}_{\mathbf{1 1}}$ can be called the computation of the odd frequency part in the transform matrix [4].

In Equation (4), the integers of matrix $\boldsymbol{T}_{\mathbf{0 0}}$ have the symmetry property, using matrix decomposition $\boldsymbol{T}_{\mathbf{0 0}}$ can be expressed as the product of the three sparse matrices $\boldsymbol{R}_{\mathbf{1}}, \boldsymbol{T}_{\mathbf{0}}$ and $\boldsymbol{R}_{\mathbf{2}}$ as was done with the original matrix $\boldsymbol{T}$ $[4,7]$. The result of the decomposition for $\boldsymbol{T}_{\mathbf{0 0}}$ is shown in Equation (5).

$$
T_{00}=R_{r} \cdot T_{0} \cdot R_{1}
$$

Where

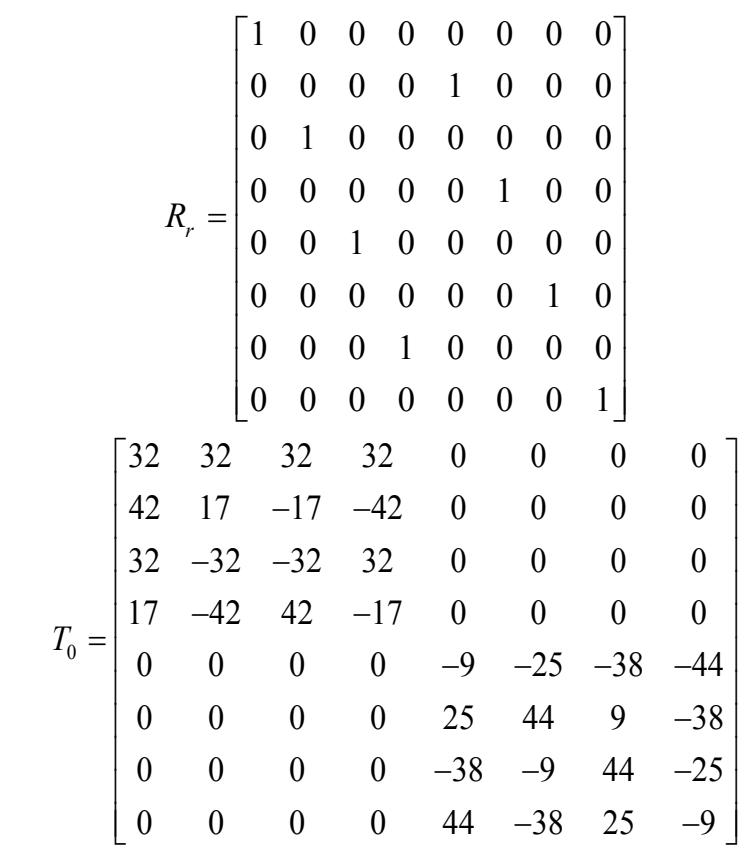

And

$$
R_{1}=\left[\begin{array}{cccccccc}
1 & 0 & 0 & 0 & 0 & 0 & 0 & 1 \\
0 & 1 & 0 & 0 & 0 & 0 & 1 & 0 \\
0 & 0 & 1 & 0 & 0 & 1 & 0 & 0 \\
0 & 0 & 0 & 1 & 1 & 0 & 0 & 0 \\
0 & 0 & 0 & -1 & 1 & 0 & 0 & 0 \\
0 & 0 & -1 & 0 & 0 & 1 & 0 & 0 \\
0 & -1 & 0 & 0 & 0 & 0 & 1 & 0 \\
-1 & 0 & 0 & 0 & 0 & 0 & 0 & 1
\end{array}\right]
$$

As shown in the above equation, $\boldsymbol{R}_{\mathbf{1}}$ can be implemented using additions and subtractions only and have a complexity of 8 additions, while $\boldsymbol{R}_{\boldsymbol{r}}$ doesn't have any complexity at all [4], as for $\boldsymbol{T}_{\mathbf{0}}$ it can be expressed by the direct sum of matrices $\boldsymbol{T}_{\boldsymbol{0}}$ and $\boldsymbol{T}_{\mathbf{0} \boldsymbol{o}}$, as shown in Equation (6).

$$
T_{0}=T_{0 E} \oplus T_{0 O}
$$

Where

$$
\begin{aligned}
& T_{0 E}=\left[\begin{array}{cccc}
32 & 32 & 32 & 32 \\
42 & 17 & -17 & -42 \\
32 & -32 & -32 & 32 \\
17 & -42 & 42 & -17
\end{array}\right] \\
& T_{0 O}=\left[\begin{array}{cccc}
-9 & -25 & -38 & -44 \\
25 & 44 & 9 & -38 \\
-38 & -9 & 44 & -25 \\
44 & -38 & 25 & -9
\end{array}\right]
\end{aligned}
$$

The symmetry of the matrix $\boldsymbol{T}_{\mathbf{0} \boldsymbol{E}}$ can be further exploited using matrix decomposition to decompose the matrix into 
the product of sparse matrices $\boldsymbol{U}_{\mathbf{1}}$ and $\boldsymbol{U}_{\mathbf{2}}$ [4], as shown in Equation (7).

$$
T_{0 E}=U_{1} \cdot U_{2}
$$

Where

$$
U_{1}=\left[\begin{array}{cccc}
32 & 32 & 0 & 0 \\
0 & 0 & -17 & -42 \\
32 & -32 & 0 & 0 \\
0 & 0 & 42 & -17
\end{array}\right]
$$

And

$$
U_{2}=\left[\begin{array}{cccc}
1 & 0 & 0 & 1 \\
0 & 1 & 1 & 0 \\
0 & -1 & 1 & 0 \\
-1 & 0 & 0 & 1
\end{array}\right]
$$

In Equation (7), $\boldsymbol{U}_{\mathbf{2}}$ can be implemented using additions and subtractions only and have a complexity of 4 additions, while $\boldsymbol{U}_{\mathbf{1}}$ can be implemented using 10 additions and 10 shifts. This would sum up to a complexity of 14 addition and 10 shift operations for Equation (7) [4].

Although there is no symmetry present in matrix $\boldsymbol{T}_{\mathbf{0}} \boldsymbol{o}$, the matrix addition can be used to segment the matrix into two matrices with more value coherence in their elements, as shown in Equation (8).

$$
\begin{gathered}
T_{0 O}=K_{0}+K_{1} \\
K_{0}=\left[\begin{array}{cccc}
-9 & -24 & -40 & -36 \\
24 & 36 & 9 & -40 \\
-40 & -9 & 36 & -24 \\
36 & -40 & 24 & -9
\end{array}\right] \\
K_{1}=\left[\begin{array}{cccc}
0 & -1 & 2 & -8 \\
1 & 8 & 0 & 2 \\
2 & 0 & 8 & -1 \\
8 & 2 & 1 & 0
\end{array}\right]
\end{gathered}
$$

After using the matrix addition, $\boldsymbol{K}_{\mathbf{0}}$ can be simplified using matrix decomposition into the product of matrices $\boldsymbol{K}_{\mathbf{2}}$ and $\boldsymbol{K}_{\mathbf{3}}$. The result of the decomposition for $\boldsymbol{K}_{\mathbf{0}}$ is shown in Equation (9).

$$
K_{0}=K_{2} \cdot K_{3}
$$

Where

$$
K_{2}=\left[\begin{array}{cccc}
1 & 0 & 0 & 4 \\
0 & 1 & 4 & 0 \\
0 & 4 & -1 & 0 \\
-4 & 0 & 0 & 1
\end{array}\right]
$$

And

$$
K_{3}=\left[\begin{array}{cccc}
-9 & 8 & -8 & 0 \\
-8 & 0 & 9 & -8 \\
8 & 9 & 0 & -8 \\
0 & -8 & -8 & -9
\end{array}\right]
$$

As shown in the above equation; $\boldsymbol{K}_{\mathbf{1}}$ can be implementted using additions and shifts only and have a complexity of 8 additions and 8 shifts, the addition between $\boldsymbol{K}_{\mathbf{1}}$ and the product of $\boldsymbol{K}_{\mathbf{2}}$ and $\boldsymbol{K}_{\mathbf{3}}$ has a complexity of 4 additions, As for the matrix $\boldsymbol{K}_{\mathbf{2}}$ it have a complexity of 4 additions and 4 shifts, while on the other hand $\boldsymbol{K}_{\mathbf{3}}$ have a complexity of 12 additions and 4 shifts. This in the end sums the complexity of $\boldsymbol{T}_{\mathbf{0}} \boldsymbol{o}$ to 28 additions and 16 shifts [4,5]. All of the above decomposition and summation sum the complexity of $\boldsymbol{T}_{\mathbf{0 0}}$ to 66 additions and 32 shifts [4,5].

Turning to matrix $T_{11}$ which represents the computation of the odd frequency part, the matrix as shown in Equation (10), doesn't have the symmetric property within its elements, in order to be able to decompose this matrix the modification techniques will be used. For the decomposition of this matrix two proposed algorithms will be presented in this paper.

\subsection{Proposed Algorithm 1}

For Proposed Algorithm 1, the odd frequency modified integer cosine transformation matrix based on the dyadic symmetry concept used by Wai-Kuen Cham in [7] is obtained by modifying the positions of the elements in the matrix to provide the matrix basic vectors with orthogonality regardless of the matrix elements values [7]. The matrix $\mathrm{T}_{11}$ and the modified matrix are shown in Equation (10) below.

$$
T_{11}:=T_{1 \mathrm{mod}}
$$

Where

$$
T_{11}=\left[\begin{array}{cccccccc}
-4 & -13 & -21 & -29 & -35 & -40 & -43 & -45 \\
13 & 35 & 45 & 40 & 21 & -4 & -29 & -43 \\
-21 & -45 & -29 & 13 & 43 & 35 & -4 & -40 \\
29 & 40 & -13 & 45 & -4 & 43 & 21 & -35 \\
-35 & -21 & 43 & 4 & -45 & 13 & 40 & -29 \\
40 & -4 & -35 & 43 & -13 & -29 & 45 & -21 \\
-43 & 29 & -4 & -21 & 40 & -45 & 35 & -13 \\
45 & -43 & 40 & -35 & 29 & -21 & 13 & -4
\end{array}\right]
$$

and

$$
T_{1 \text { mod }}=\left[\begin{array}{cccccccc}
-4 & -13 & -21 & -29 & -35 & -40 & -43 & -45 \\
35 & 40 & 43 & 45 & -4 & -13 & -21 & -29 \\
-21 & -29 & 4 & 13 & 43 & 45 & -35 & -40 \\
45 & 43 & -40 & -35 & 29 & 21 & -13 & -4 \\
-43 & -45 & 35 & -40 & -21 & 29 & 4 & -13 \\
13 & -4 & -29 & 21 & -40 & 35 & 45 & -43 \\
-29 & 21 & -13 & 4 & 45 & -43 & 40 & -35 \\
40 & -35 & 45 & -43 & 13 & -4 & 29 & -21
\end{array}\right]
$$

Replacing $\boldsymbol{T}_{\mathbf{1 1}}$ with $\boldsymbol{T}_{\mathbf{1} \bmod }$ as the new odd frequency transformation matrix [7], this matrix can be segmented into two matrices with more value coherence in their 
elements as was done in the matrix $\boldsymbol{T}_{\mathbf{0 0}}$, as shown in Equation (11).

$$
T_{1 \mathrm{mod}}=T_{m 1}+T_{m 2}
$$

Where

$$
T_{m 1}=\left[\begin{array}{cccccccc}
-4 & -16 & -24 & -32 & -36 & -44 & -44 & -44 \\
36 & 44 & 44 & 44 & -4 & -16 & -24 & -32 \\
-24 & -32 & 4 & 16 & 44 & 44 & -36 & -44 \\
44 & 44 & -44 & -36 & 32 & 24 & -16 & -4 \\
-44 & -44 & 36 & -44 & -24 & 32 & 4 & -16 \\
16 & -4 & -32 & 24 & -44 & 36 & 44 & -44 \\
-32 & 24 & -16 & 4 & 44 & -44 & 44 & -36 \\
44 & -36 & 44 & -44 & 16 & -4 & 32 & -24
\end{array}\right]
$$

$$
\text { and } T_{m 2}=\left[\begin{array}{cccccccc}
0 & 3 & 3 & 3 & 1 & 4 & 1 & -1 \\
-1 & -4 & -1 & 1 & 0 & 3 & 3 & 3 \\
3 & 3 & 0 & -3 & -1 & 1 & 1 & 4 \\
1 & -1 & 4 & 1 & -3 & -3 & 3 & 0 \\
1 & 1 & -1 & 4 & 3 & -3 & 0 & 3 \\
-3 & 0 & 3 & -3 & 4 & -1 & 1 & 1 \\
3 & -3 & 3 & 0 & 1 & 1 & -4 & 1 \\
-4 & 1 & 1 & 1 & -3 & 0 & -3 & 3
\end{array}\right]
$$

In Equation (11), the segment $\boldsymbol{T}_{\boldsymbol{m} \mathbf{1}}$ from the matrix addition segmentation done on the matrix $\boldsymbol{T}_{\mathbf{1} \text { mod }}$ has symmetric properties; hence it can be further simplified using matrix decomposition algorithm into the product of three sparse matrices [7]. The result of this matrix decomposition is shown in Equation (12).

$$
\begin{aligned}
T_{m 1}=M_{1} \cdot M_{2} \cdot M_{3} \cdot 4 & \\
\text { Where } M_{1} & =\left[\begin{array}{cccccccc}
-2 & 0 & 1 & -1 & -1 & 3 & -1 & 0 \\
3 & -1 & 1 & 1 & 0 & 2 & 0 & 1 \\
-1 & -3 & 1 & 0 & 1 & 0 & 2 & -1 \\
0 & 1 & 0 & 1 & 3 & 1 & -1 & -2 \\
1 & -1 & -3 & -2 & 0 & 1 & 0 & -1 \\
1 & 1 & 1 & 0 & -2 & 0 & 1 & -3 \\
0 & -2 & 0 & 1 & -1 & -1 & -3 & -1 \\
-1 & 0 & -2 & 3 & -1 & 1 & 1 & 0
\end{array}\right] \\
M_{2} & =\left[\begin{array}{cccccccc}
0 & 0 & 0 & 0 & 1 & -1 & 0 & 1 \\
0 & 0 & 1 & 1 & 0 & 0 & 1 & 0 \\
0 & -1 & 0 & -1 & 0 & 0 & 1 & 0 \\
0 & -1 & 1 & 0 & 0 & 0 & -1 & 0 \\
0 & -1 & -1 & 1 & 0 & 0 & 0 & 0 \\
-1 & 0 & 0 & 0 & 0 & -1 & 0 & -1 \\
-1 & 0 & 0 & 0 & -1 & 0 & 0 & 1 \\
-1 & 0 & 0 & 0 & 1 & 1 & 0 & 0
\end{array}\right]
\end{aligned}
$$

and

$$
M_{3}=\left[\begin{array}{cccccccc}
-1 & 1 & -2 & 0 & 1 & 0 & 2 & 0 \\
-2 & 0 & 1 & -1 & -2 & 0 & 1 & 0 \\
2 & -1 & 0 & 0 & -1 & -1 & 2 & 0 \\
1 & 1 & 0 & -2 & 0 & 1 & 0 & 2 \\
0 & 2 & 1 & 1 & 0 & -2 & 0 & 1 \\
-1 & -2 & 0 & 0 & 1 & -1 & 0 & 2 \\
0 & 0 & -2 & 1 & -2 & 0 & -1 & 1 \\
0 & 0 & 1 & 2 & 0 & 2 & 1 & 1
\end{array}\right]
$$

As shown from the equation above; $\boldsymbol{M}_{\mathbf{1}}$ can be implemented using additions and shifts only and have a complexity of 48 additions and 8 shifts, while $\boldsymbol{M}_{\mathbf{2}}$ can be implemented using only additions and have a complexity of 16 additions, As for the matrix $\boldsymbol{M}_{3}$ it have a complexity of 32 additions and 8 shifts. This in the end sums the complexity of $\boldsymbol{T}_{\boldsymbol{m} \mathbf{1}}$ to 96 additions and 40 shifts [7]. On the other hand, $\boldsymbol{T}_{\boldsymbol{m} \mathbf{2}}$ can be implemented using additions and shifts only, and has a complexity of 72 additions and 8 shifts $[4,5,7]$.

By using equations from Equation (2) to Equation (12), the efficient fast multiplication-free integer transformation for the 2-D DCT matrix for the H.265 standard for proposed algorithm 1 is given as shown in Equation (13).

$$
\begin{aligned}
T=P_{r} \cdot\left\{\left[R_{r} \cdot\left[\left(U_{1} \cdot U_{2}\right) \oplus\left(K_{1}+\left(K_{2} \cdot K_{3}\right)\right)\right] \cdot R_{1}\right] \oplus\right. \\
{\left.\left[T_{m 1}+\left(M_{1} \cdot M_{2} \cdot M_{3} \cdot 4\right)\right]\right\} \cdot P_{1} }
\end{aligned}
$$

\subsection{Proposed Algorithm 2}

In the proposed algorithm presented in this section the same complexity reduction and decomposition techniques will be done, the difference will be that in the odd frequencies matrix in the step done in Equation (10) instead of only using dyadic symmetry to rearrange the elements of the matrix, a modification in values of the elements will be done so that it matches the matrix Tm1 in Equation (11). The resultant odd frequency matrix is given in Equation (14).

$$
T_{11}:=T_{1 \bmod 2}
$$

Where

$$
T_{1 \bmod 2}=\left[\begin{array}{cccccccc}
-4 & -16 & -24 & -32 & -36 & -44 & -44 & -44 \\
36 & 44 & 44 & 44 & -4 & -16 & -24 & -32 \\
-24 & -32 & 4 & 16 & 44 & 44 & -36 & -44 \\
44 & 44 & -44 & -36 & 32 & 24 & -16 & -4 \\
-44 & -44 & 36 & -44 & -24 & 32 & 4 & -16 \\
16 & -4 & -32 & 24 & -44 & 36 & 44 & -44 \\
-32 & 24 & -16 & 4 & 44 & -44 & 44 & -36 \\
44 & -36 & 44 & -44 & 16 & -4 & 32 & -24
\end{array}\right]
$$

The odd frequency matrix for proposed algorithm 2 $\boldsymbol{T}_{\mathbf{1 m o d 2}}$ can be decomposed for complexity reduction in 
the same manner as done in Equation (12) in Subsection 3.1. This means that the two proposed algorithm have exactly the same decomposition and segmentation, the only exception is that the odd frequency part for proposed algorithm 2 consists of $\boldsymbol{T}_{\boldsymbol{m} \mathbf{1}}$ only while for proposed algorithm 1 it consists of the addition of $\boldsymbol{T}_{\boldsymbol{m} 1}$ and $\boldsymbol{T}_{\boldsymbol{m} 2}$.

By using equations from Equation (2) to Equation (14), the efficient fast multiplication-free integer transformation for the 2-D DCT matrix for the H.265 standard for proposed algorithm 2 is given as shown in Equation (15).

$$
\begin{gathered}
T=P_{r} \cdot\left\{\left[R_{r} \cdot\left[\left(U_{1} \cdot U_{2}\right) \oplus\left(K_{1}+\left(K_{2} \cdot K_{3}\right)\right)\right] \cdot R_{1}\right] \oplus\right. \\
\left.\left[\left(M_{1} \cdot M_{2} \cdot M_{3} \cdot 4\right)\right]\right\} \cdot P_{1}
\end{gathered}
$$

For the proposed algorithms and the original algorithm, the complexity evaluation is done by calculating the number of additions, shifts and multiplications needed to implement it. The complexity evaluation summary for the proposed and original algorithms is shown in Table 1.

\section{Analysis and Comparison of Transformation Quality}

In order to test the efficiency of the proposed algorithms, evaluation of the quality of the reconstructed video compared to the original video is done using the quality assessment metrics; the three quality metrics used in this paper are the MSE, PSNR and the SSIM. The tests done in this section are applied to standard high definition video quality assessment sequences as developed by Dr. Karl Mauthe at Taurus Media Technik. The full description of the test sequences used is shown in Table 2.

Using the Matlab computational tool the quality metrics for original and the proposed algorithms were calculated for 100 frames of the four different standard test sequences. The aim is to evaluate the algorithms quality and reliability, and determine the efficiency of each of the proposed algorithms compared to the original.
Table 1. Complexity Evaluation Comparison table for Original and Proposed Algorithms

\begin{tabular}{lllll}
\hline & \multicolumn{4}{c}{ Complexity } \\
\hline Operation & $\begin{array}{l}\text { Original } \\
\text { Algorithm }\end{array}$ & $\begin{array}{l}\text { Proposed Algo- } \\
\text { rithm 1 }\end{array}$ & $\begin{array}{l}\text { Proposed } \\
\text { gorithm 2 }\end{array}$ & Al- \\
Additions & 240 & 242 & 162 \\
Multiplications & 256 & 0 & 0 \\
Shifts & 0 & 58 & 50 \\
\hline
\end{tabular}

Table 2. Test Sequences Information

\begin{tabular}{lll}
\hline Sequence & \#Frames & Short Description \\
\hline Blue sky & 250 & $\begin{array}{l}\text { Top of two trees against blue sky. } \\
\text { High contrast, small color differences } \\
\text { in the sky, many details. Camera rota- } \\
\text { tion. } \\
\text { Shot of a pedestrian area. Low camera } \\
\text { position, people pass by very close to } \\
\text { the camera. High depth of field. Static } \\
\text { camera. } \\
\text { Redestrian Area }\end{array}$ \\
& 250 & $\begin{array}{l}\text { Riverbed seen through the water. Very } \\
\text { hard to code. } \\
\text { View from a bridge to Munich station. } \\
\text { Evening shot. Long zoom out. Many } \\
\text { details, regular structures (tracks). }\end{array}$ \\
Riverbed & 313 &
\end{tabular}

The quality metrics results of the quality assessment for the Blue Sky sequence are shown in the Figures 1, 2 and 3.

The quality metrics results of the quality assessment for the Pedestrian Area sequence are shown in the Figures 4, 5 and 6.

The quality metrics results of the quality assessment for the Riverbed sequence are shown in the Figures 7, 8 and 9.

The quality metrics results of the quality assessment for the Station_2 sequence are shown in the Figures 10, 11 and 12.

The figures from 1 to 12 show all the test results done to evaluate the quality of the proposed algorithms compared to the original algorithm, all these results are

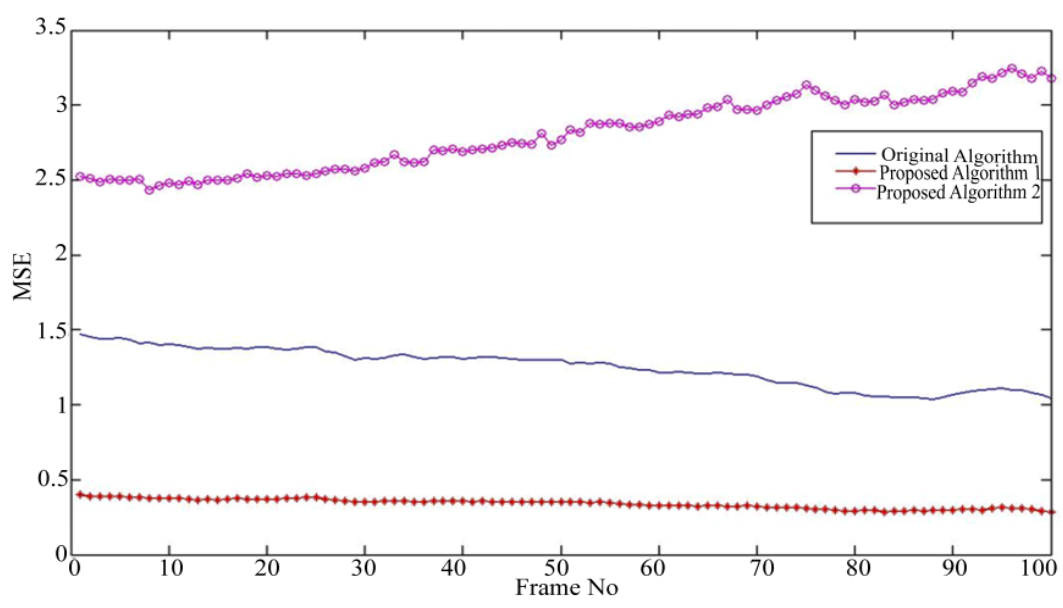

Figure 1. Mean MSE for Original and Proposed Algorithms 


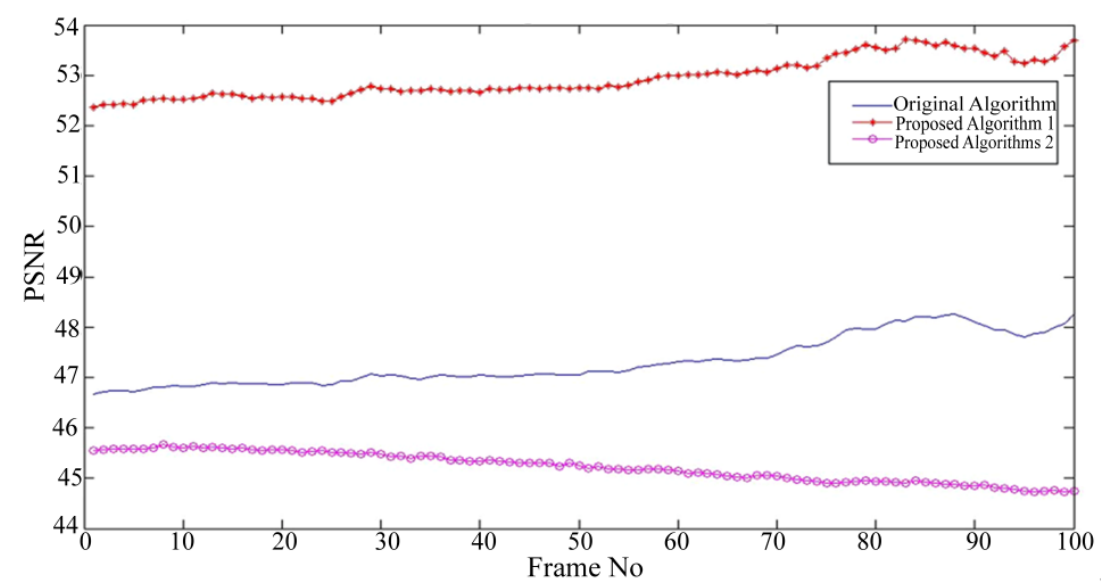

Figure 2. Mean PSNR for Original and Proposed Algorithms

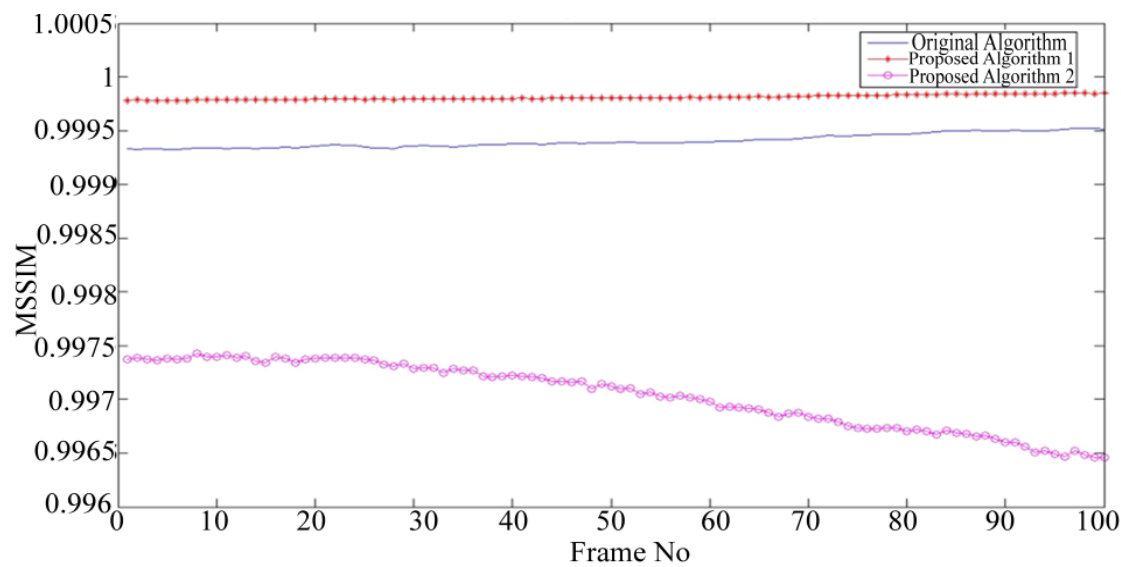

Figure 3. Mean SSIM for Original and Proposed Algorithms

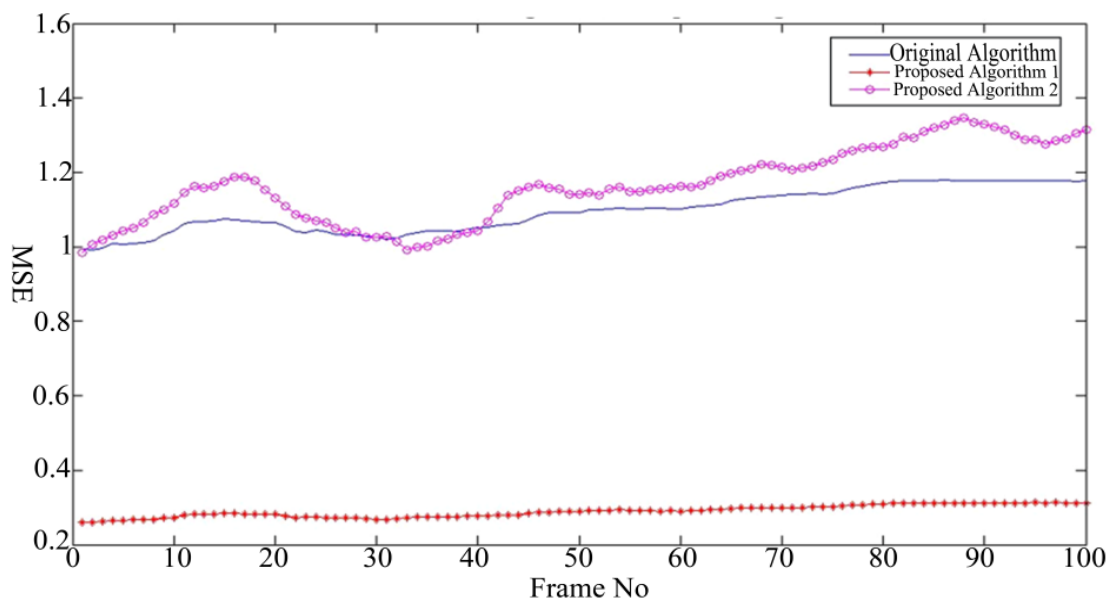

Figure 4. Mean MSE for Original and Proposed Algorithms

summarized and combined with the complexity of all the algorithms to determine the efficiency of the proposed algorithms. Tables 3, 4 and 5 show the summarized results for the MSE, PSNR and the MSSIM respectively.

\section{Comparison of Computational Complexity and Quality Evaluation}

In this section, evaluation of the proposed algorithms versus the original algorithm is done through a comparison 


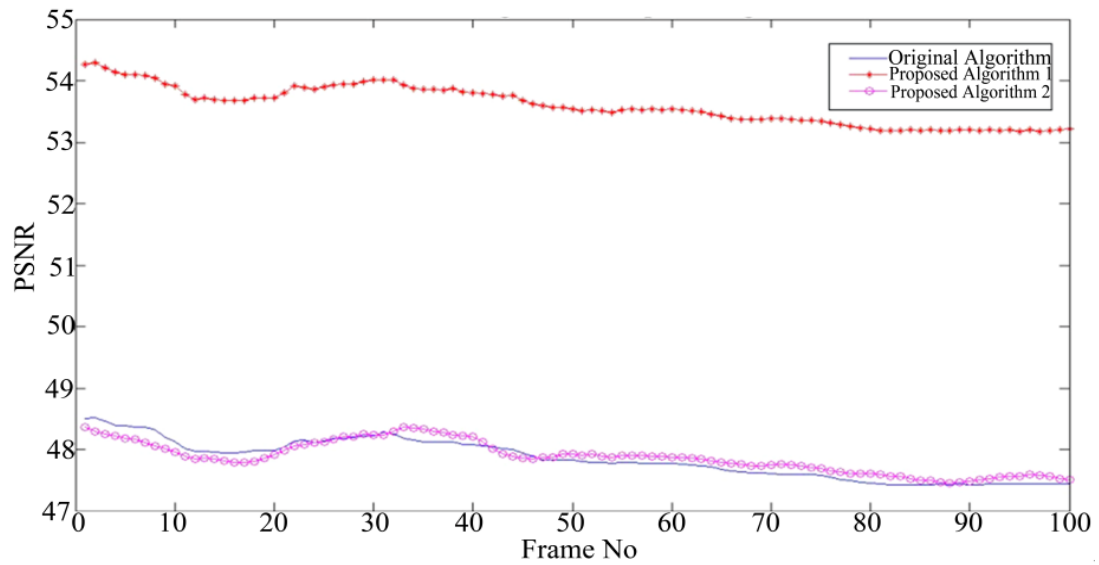

Figure 5. Mean PSNR for Original and Proposed Algorithms

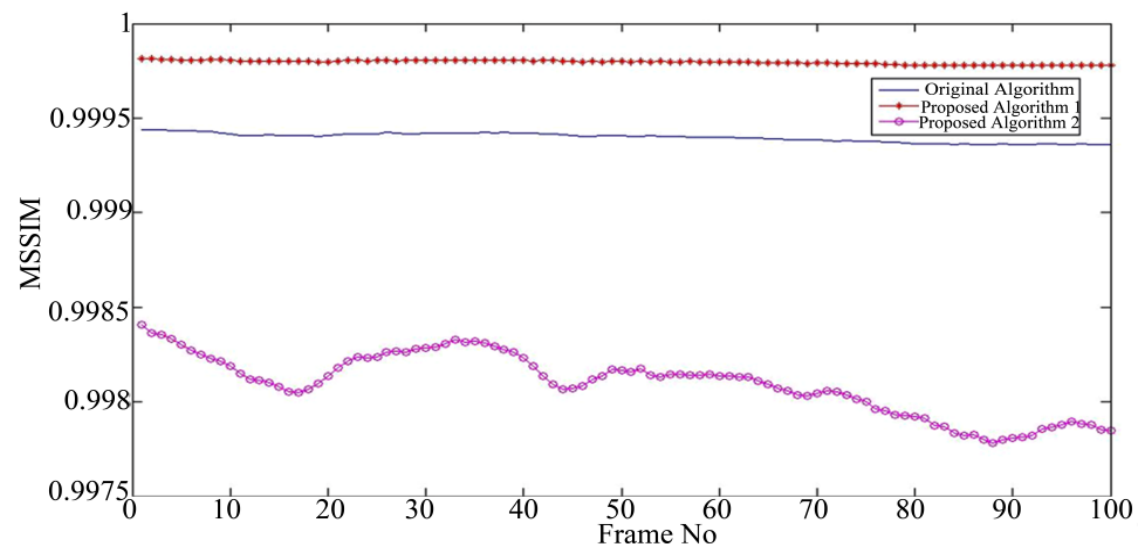

Figure 6. Mean SSIM for Original and Proposed Algorithms

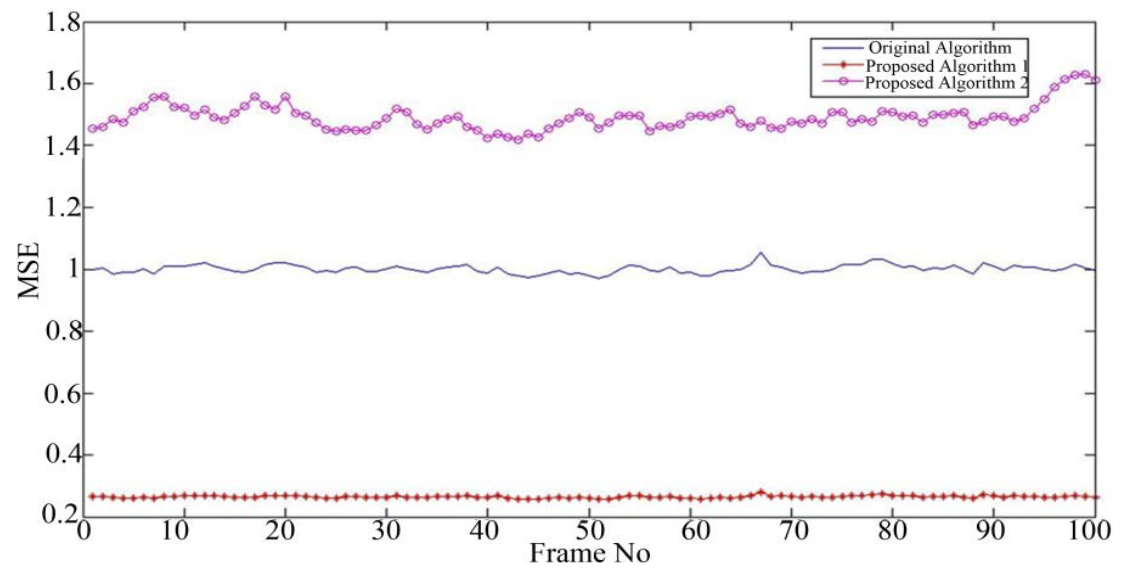

Figure 7. Mean MSE for Original and Proposed Algorithms

Table 3. Average MSE Comparison table for Original and Proposed Algorithms

\begin{tabular}{llll}
\hline & Average MSE & & Proposed Algorithm 2 \\
\hline Sequence & Original Algorithm & Proposed Algorithm 1 & 2.8050 \\
Blue_Sky & 1.2513 & 0.3399 & 1.1636 \\
Pedestrian_Area & 1.0966 & 0.2893 & 1.4918 \\
Riverbed & 1.0009 & 0.2646 & 1.0008 \\
Station & 0.9314 & 0.2449 & \\
\hline
\end{tabular}


Table 4. Average PSNR Comparison table for Original and Proposed Algorithms

\begin{tabular}{llll}
\hline & Average PSNR & \\
\hline Sequence & Original Algorithm & Proposed Algorithm 1 & Proposed Algorithm 2 \\
Blue_Sky & 47.3093 & 52.9384 & 45.2233 \\
Pedestrian_Area & 47.8787 & 53.5946 & 47.8456 \\
Riverbed & 48.2731 & 54.0194 & 47.3802 \\
Station & 49.0446 & 54.7329 & 48.3484 \\
\hline
\end{tabular}

Table 5. Average SSIM Comparison table for Original and Proposed Algorithms

\begin{tabular}{llll}
\hline & Average MSSIM & & \\
\hline Sequence & Original Algorithm & Proposed Algorithm 1 & Proposed Algorithm 2 \\
Blue_Sky & 0.9994 & 0.9998 & 0.9970 \\
Pedestrian_Area & 0.9994 & 0.9998 & 0.9981 \\
Riverbed & 0.9995 & 0.9998 & 0.9976 \\
Station & 0.9995 & 0.9998 & 0.9982 \\
\hline
\end{tabular}

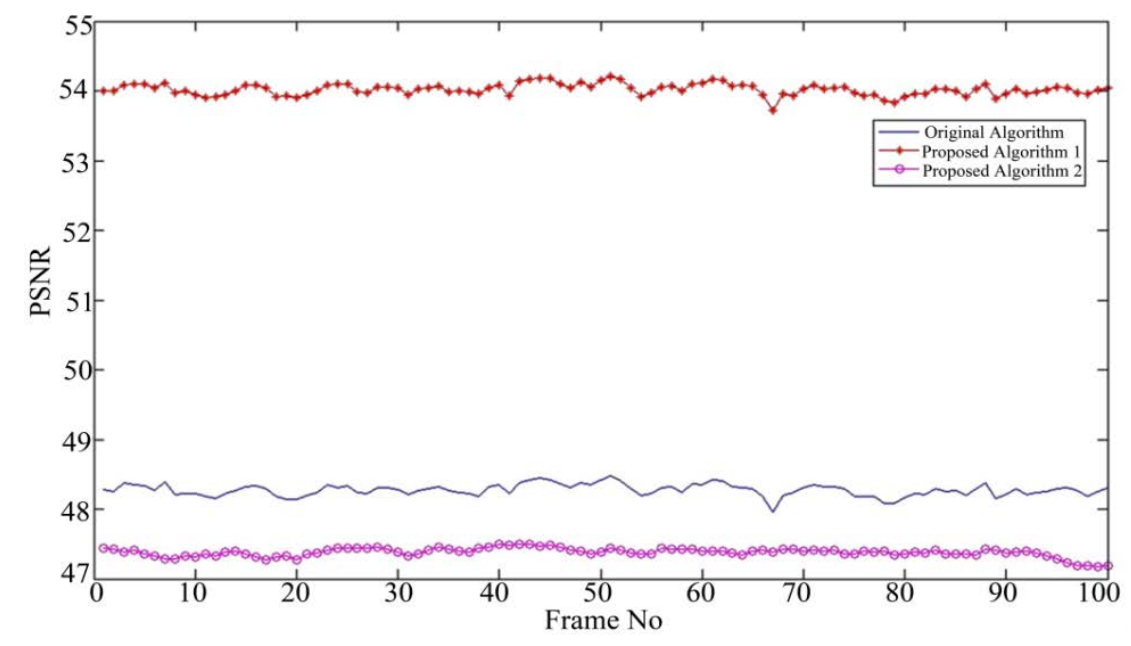

Figure 8. Mean PSNR for Original and Proposed Algorithms

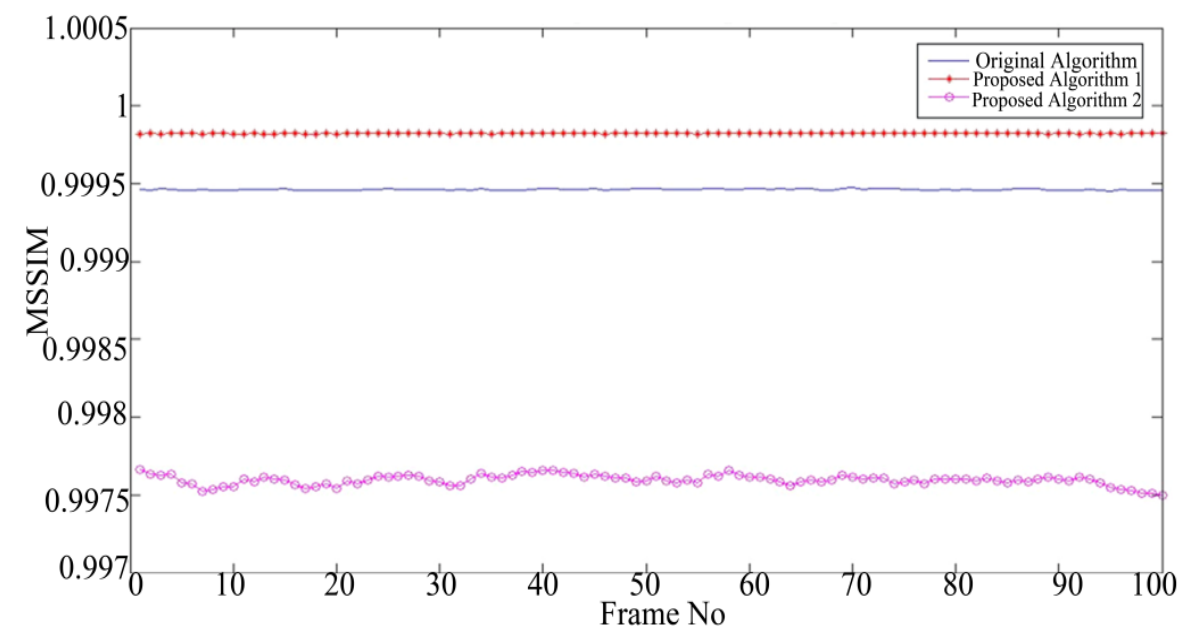

Figure 9. Mean SSIM for Original and Proposed Algorithms 


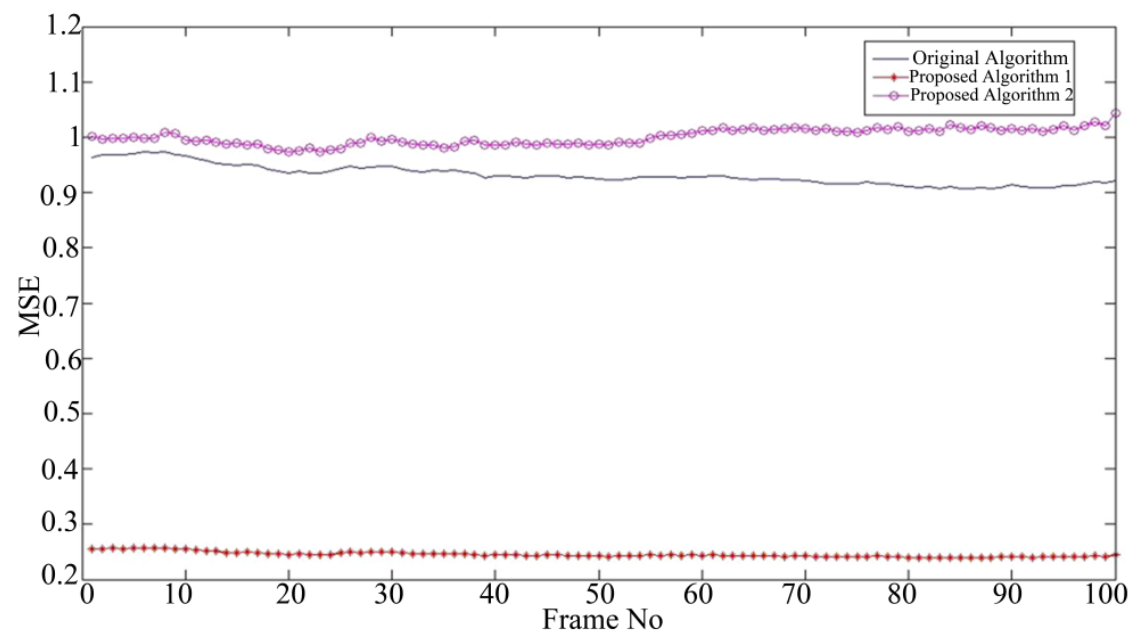

Figure 10. Mean MSE for Original and Proposed Algorithms

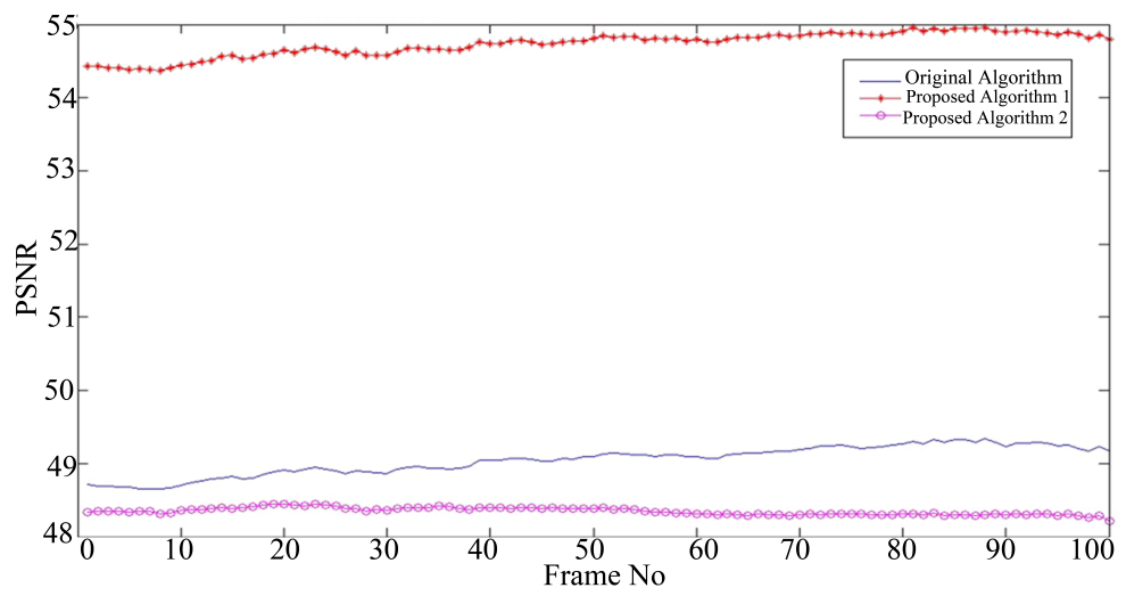

Figure 11. Mean PSNR for Original and Proposed Algorithms

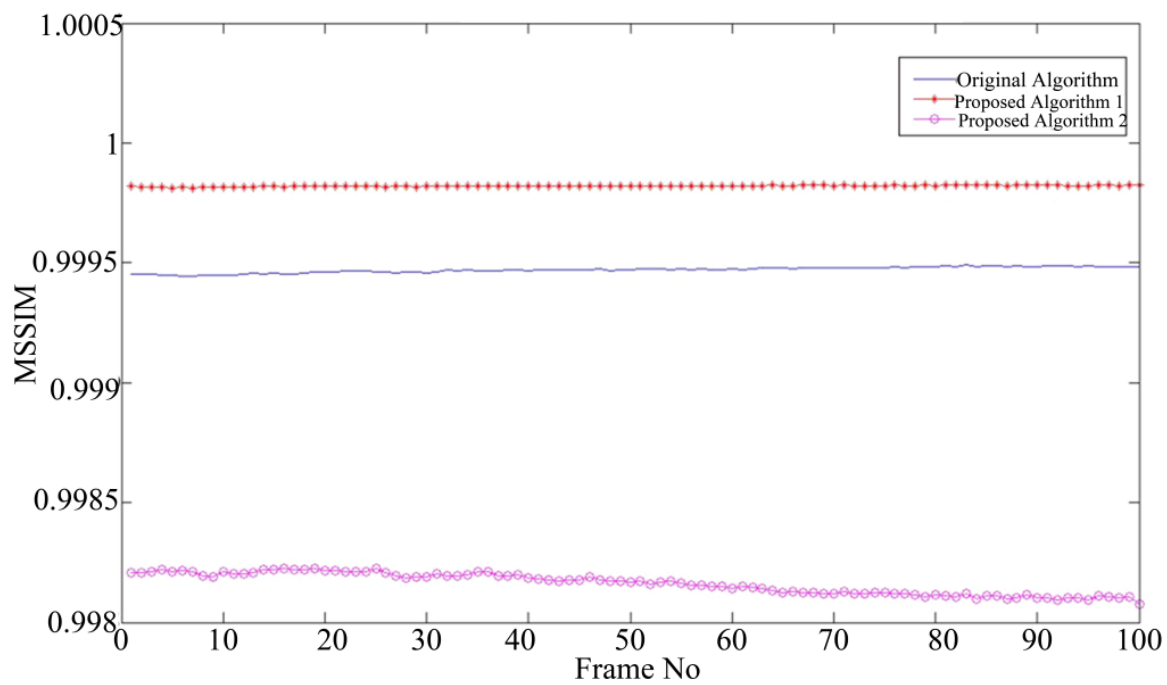

Figure 12. Mean SSIM for Original and Proposed Algorithms 
between the results of Section 3 and Section 4 .

In terms of quality assessment Table 3 clearly shows the advantage of Proposed Algorithm 1 over Proposed Algorithm 2 and even the Original algorithm as it was able to achieve the smallest values for the MSE index over 4 different test sequences. Table 4 backs the results shown in Table 3, and also shows that the different in quality measured by the PSNR index between the originnal algorithm and Proposed Algorithm 2 is not high, which means that both achieve comparable qualities. Table 5 shows that according to a more (HVS) based index, Proposed Algorithm 1 still achieve the best result with the original algorithm coming in second place, and proposed algorithm achieving lower structural similarity than both. However the values in this table indicate that the results for the three algorithms are all almost in the same range, and achieve what is considered high structural similarity. These improved results achieved by the proposed algorithms compared to the original one are due to applying the dyadic symmetry on the transformation matrix odd-frequency part which makes the transformation matrix as a whole symmetric and hence eliminates the transformation error as the product of the transformation matrix multiplied by its transpose will yield an identity matrix.

As for the complexity point of view, taking into consideration that multiplications are the process in terms of complexity and hardware followed by additions and finally shifts. Table 1 clearly shows the superiority of proposed Algorithm 2 over the other two algorithms in terms of less complexity, and emphasis the fact that both of the proposed algorithms are multiplication-free, also the table shows that proposed Algorithm 1 is far less complex than the original algorithm, although it requires two more additions than the original algorithm, it has no multiplications and small number of shifts.

Finally form all the results obtained in this section, it can be concluded that the proposed Algorithm 1 achieves the highest quality in encoding and decoding while maintaining less complexity than the original algorithm, which means that it would be suitable for quality-oriented applications, however on the other hand proposed Algorithm 2 achieves the dramatically less complexity than the original algorithm without having any noticeable or detectable quality degradation, which makes this algorithm suitable for speed or hardware oriented applications.

\section{Conclusions}

A new $16 \times 16$ DCT matrix was recently introduced for the highly anticipated H.265 standard, this DCT matrix is developed for high definition videos encoding and decoding, the aim is to make them less complex and faster for video communication and transmission, like in high definition broadcasting and storage. Two new algorithms were proposed in this paper. The first technique is a quality oriented algorithm while offering multiplication-free complexity. The second algorithm is a complexity and speed oriented algorithm while maintaining almost the same quality offered by the original algorithm.

The aim of proposing an efficient fast 1-D algorithm for this DCT matrix is to reduce the complexity and hence the hardware and increase the speed of computation to meet the constantly improving demands in the fields of communication and transmission. Quality Assessment Tests were carried out and the quality metrics MSE, PSNR and SSIM were calculated to evaluate the performance of the proposed algorithms compared to the original one. The test results showed that the first proposed algorithm offers better quality, objective and subjective, while offering less complexity and multiplication-free computation. While the second proposed algorithm offers almost the same quality, objective and subjective, while offering much less complexity and multiplication-free computation than the original algorithm and the first proposed one.

\section{REFERENCES}

[1] J.-B. Lee and H. Kalva, "The VC-1 and H.264 Video Compression Standards for Broadband Video Services," Springer, New York, 2008.

[2] H. 264/MPEG-4 Part 10: Overview. http://www.vcodex. com/files/h264_overview_orig.pdf

[3] http://www.h265.net/index.php?s=det

[4] C.-P. Fan and G.-A. Su, "Efficient Fast 1-D $8 \times 8$ Inverse Integer Transform for VC-1 Application," IEEE Transactions on Circuits and Systems for Video Technology, Vol. 19, No. 4, 2009, pp. 584-590.

[5] C.-P. Fanm and G.-A. Su, "Efficient Low-Cost Sharing Design of Fast 1-D Inverse Integer Transform Algorithms for H.264/AVC and VC-1," IEEE Signal Processing Letters, Vol. 15, 2008, pp. 926-929.

[6] W.-K. Cham, "Development of Integer Cosine Transformation by the Principle of Dyadic Symmetry," IEEE Proceedings, Vol. 136, No. 4, August 1989, pp. 276-282.

[7] J. Dong, K. N. Ngan, C.-K. Fong and W.-K. Cham, "2D Order-16 Integer Transforms for HD Video Coding," IEEE Transactions on Circuits and Systems for Video Technology, Vol. 19, No. 10, October 2009, pp. 14631474. 